\title{
Differentiation and Molecular Properties of Mesenchymal Stem Cells Derived from Murine Induced Pluripotent Stem Cells Derived on Gelatin or Collagen
}

\author{
Chizuka Obara, Kazuya Takizawa, Kenichi Tomiyama, Masaharu Hazawa, \\ Ai Saotome-Nakamura, Takaya Gotoh, Takeshi Yasuda, and Katsushi Tajima
}

Research Center for Radiation Emergency Medicine, National Institute of Radiological Sciences, 4-9-1 Anagawa, Inage-ku, Chiba-shi, Chiba 263-8555, Japan

Correspondence should be addressed to Katsushi Tajima; tajima@nirs.go.jp

Received 2 February 2016; Revised 9 July 2016; Accepted 19 July 2016

Academic Editor: Pavla Jendelova

Copyright (C) 2016 Chizuka Obara et al. This is an open access article distributed under the Creative Commons Attribution License, which permits unrestricted use, distribution, and reproduction in any medium, provided the original work is properly cited.

\begin{abstract}
The generation of induced-pluripotential stem cells- (iPSCs-) derived mesenchymal stem cells (iMSCs) is an attractive and promising approach for preparing large, uniform batches of applicable MSCs that can serve as an alternative cell source of primary MSCs. Appropriate culture surfaces may influence their growth and differentiation potentials during iMSC derivation. The present study compared molecular properties and differentiation potential of derived mouse iPS-MSCs by deriving on gelatin or collagencoated surfaces. The cells were derived by a one-step method and expressed CD73 and CD90, but CD105 was downregulated in iMSCs cultured only on gelatin-coated plates with increasing numbers of passages. A pairwise scatter analysis revealed similar expression of MSC-specific genes in iMSCs derived on gelatin and on collagen surfaces as well as in primary mouse bone marrow MSCs. Deriving iMSCs on gelatin and collagen dictated their osteogenic and adipose differentiation potentials, respectively. Derived iMSCs on gelatin upregulated Bmp2 and Lif prior to induction of osteogenic or adipose differentiation, while PPAR $\gamma$ was upregulated by deriving on collagen. Our results suggest that extracellular matrix components such as gelatin biases generated iMSC differentiation potential towards adipose or bone tissue in their derivation process via up- or downregulation of these master genes.
\end{abstract}

\section{Introduction}

Mesenchymal stem cell- (MSC-) mediated cytotherapy has attracted increasing interest owing to its safety and effectiveness in a number of auto-, allo-, and xenogeneic animal models $[1,2]$. MSCs can be easily harvested from various adult human tissues and rapidly expanded in vitro, although this may limit their properties such as plasticity over the long term [3]. In addition, starting tissues such as bone marrow and adipose as well as culture conditions affect MSC properties and preparation, making it difficult to compare results from different MSC studies [4]. Induced pluripotent stem cells (iPSCs) are one of the most appropriate basic cell sources to not only generate standardized cell lines but also progenitor cells [5]. Therefore, iPSCs-derived mesenchymal stem cells (iMSCs) are considered as a promising alternative cell source of primary MSCs [6]. Indeed, iMSCs proliferate extensively in vitro, possibly resolving the challenge of generating large batches of identical primary MSCs [7].

Extracellular components in the stem cell niche define the stem cell function and specification by generating signals for survival, proliferation, and differentiation; however, the underlying mechanisms are only partially understood. Therefore, it is crucial to investigate how these components affect their properties $[8,9]$. A previous report found that gelatincoating on plates increased proliferation in bone marrowderived MSCs (BMMSCs) as compared to cells cultured on uncoated plates [10]. Several reports have shown that iMSC characteristics such as differentiation potentials in vitro and in vivo are similar to those of primary progenitor cells [11], but it is not known whether extracellular components such as gelatin and collagen significantly affect iMSC characteristics 
in its derivation process. Although several studies have successfully reported the derivation of human iMSCs from iPSCs [3, 12-14], a simple and efficient method for inducing mouse iMSCs has not yet been established $[15,16]$. However, the safety of further clinical applications must be evaluated in appropriate animal models including genetically modified mouse, which requires the establishment of simple and effective derivation method for mouse iMSCs in addition to human cells.

In the present study, we designed culture plates-coated with gelatin or collagen and derived iMSCs from mouse iPSCs using these plates. After their derivation, we investigated whether the derived iMSC differentiation potential is dictated towards bone or adipose tissue using differentiation assay with uncoated plates.

\section{Materials and Methods}

2.1. Mouse Induced Pluripotent Stem Cells (iPSCs) Culture. Three mouse iPS cell (iPSC) lines (2A-4F-60, 2A-4F-100, and 2A-4F-136) were kindly gifted by Dr. Araki and Dr. Abe [17]. Cells were maintained on mouse embryonic fibroblast (MEF) in basal medium consisting of Dulbecco's modified Eagle's medium (DMEM) with 15\% knockout serum replacement, $2 \mathrm{mM}$ nonessential amino acids (NEAA), $2 \mathrm{mM} \mathrm{L-}$ glutamine (all from Invitrogen, Carlsbad, CA, USA), $0.1 \mathrm{mM}$ 2-mercaptoethanol, and leukemia inhibitory factor (LIF; 500 U/mL, ESGRO, Merck Millipore, Billerica, MA, USA).

2.2. MSC Isolation and Culture. Prospectively isolated BMMSCs over mouse bone marrow were prepared as previously described $[18,19]$. BMMSCs were isolated from three $\mathrm{C} 57 \mathrm{Bl} / 6$ mice (5 to 9 weeks old), which were purchased from Japan SLC Inc. (Shizuoka, Japan). In brief, femur and tibiae were removed, cleaned, and cut into fine pieces. The bone fragments were incubated for $1 \mathrm{~h}$ at $37^{\circ} \mathrm{C}$ in DMEM in the presence of $10 \mathrm{mM}$ HEPES, $0.2 \%$ collagenase (Wako Pure Chemical Industries, Osaka, Japan), and $5 \mathrm{U} / \mathrm{mL}$ DNase I (Takara Bio, Shiga, Japan). After digestion, they were filtered through a $70-\mu \mathrm{m}$ cell strainer (BD Falcon, Franklin Lakes, NJ) and centrifuged at $1200 \mathrm{rpm}(270 \mathrm{~g})$ for $10 \mathrm{~min}$ in a Kubota rotor RS-720 (Kubota, Tokyo, Japan). The cell pellet was resuspended in $1 \mathrm{~mL}$ of sterile distilled water for $6 \mathrm{~s}$ to disrupt the red blood cells, and then $1 \mathrm{~mL}$ of $2 \mathrm{x}$ Dulbecco's phosphate-buffered saline (D-PBS; Nissui Pharmaceutical, Tokyo, Japan) containing 4\% fetal bovine serum (FBS; HyClone, GE Healthcare Life Sciences, UT, USA) was added. The cell suspension was filtered through a cell strainer and centrifuged at $270 \mathrm{~g}$ for $5 \mathrm{~min}$. The pellet was resuspended in fresh Hank's Balanced Salt Solution containing $10 \mathrm{mM}$ HEPES, 2\% heat-inactivated fetal bovine serum (FBS) (HyClone, GE Healthcare Life Sciences, Logan, UT, USA), and 1\% penicillin/streptomycin (HBSS+) and then stained with allophycocyanin-conjugated PDGFR $\alpha$ fluorescein isothiocyanate-conjugated Sca-1, phycoerythrinconjugated CD45, and phycoerythrin-conjugated TER-119 (all from eBiosciences, San Diego, CA, USA) for $30 \mathrm{~min}$ at $4^{\circ} \mathrm{C}$. The cells were washed with $\mathrm{HBSS}+$, stained with 7-aminoactinomycin D (Beckman Coulter, Fullerton, CA, USA) to exclude dead cells, and sorted using the FACSAria cell sorter (Becton Dickinson; now BD Biosciences, San Jose, CA, USA). The fraction containing MSCs (Sca$1^{+} / \mathrm{PDGFR} \alpha^{+} / \mathrm{CD} 45^{-} /$Ter $\left.119^{-}\right)$was sorted by adequate cell gating.

The isolated MSCs were cultured in DMEM supplemented with $10 \%$ FBS and antibiotics. When the cells reached $\sim 70 \%$ confluence, they were passaged using $0.25 \%$ trypsin/EDTA (Thermo Fisher Scientific). Expanded MSCs were passaged $4(\mathrm{P} 4)$ to 7 times and used for all experiments.

\subsection{Derivation of Induced Mesenchymal Stem Cells (iMSCs)} from iPSCs Using Gelatin or Collagen-Coated Plates. We applied a modified one-step method for the derivation of mouse iMSCs based on a previously described method for human iMSCs (Figure 1) [3]. Briefly, mouse iPSCs were harvested with $0.05 \%$ trypsin/EDTA and dissociated into single cells that were seeded in the gelatin or collagen-coated plates in $10 \mathrm{~cm}$ diameter (Iwaki; AGC TECHNO GLASS Co., Ltd., Shizuoka, Japan), at a density of $15,000 / \mathrm{cm}^{2}$ in the basal medium. MEF cells were excluded from iPSCs treated with removal microbeads conjugated with antifeeder antibody (Miltenyi Biotec, Tokyo, Japan) (Catalog \#130-095-531) in every derivation experiments. In brief, mixture solution of iPSCs and MEF cells were adjusted to more than $1 \times 10^{7}$ cells in $80 \mu \mathrm{L}$ and incubated with $20 \mu \mathrm{L}$ Feeder Removal Microbeads at $4^{\circ} \mathrm{C}$ for $20 \mathrm{~min}$. After incubation, the solution which was diluted by $500 \mu \mathrm{L}$ with 5\% FBS in DMEM and was collected after being thrown into LS Columns (Miltenyi Biotec) (Catalog \#130-042-401) to remove MEF cells. The collagen-coated plates (Iwaki) (Catalog \#4020-010) were made of dried pepsin-soluble type I collagen extracted from pig tendons. The gelatin-coated plates (Iwaki) (Catalog \#4020-020) were made of dried gelatin extracted from pig skins. After $24 \mathrm{~h}$, the basal medium was supplemented with an equal volume of the derivation medium comprising basal alpha-minimum essential medium ( $\alpha$-MEM) (Invitrogen), 10\% FBS (Vitromex, San Antonio, TX, USA), $100 \mathrm{nM}$ dexamethasone, and $50 \mu \mathrm{M}$ magnesium L-ascorbic acid phosphate (all from Sigma-Aldrich, St Louis, MO, USA). After 2 days, the medium was completely replaced with the derivation medium without ROCK inhibitor, which was used in derivation of human iMSCs [3], and 8 days later, cells were harvested, thoroughly dissociated, and designated as passage zero ( 0 ). Single cells were cultured in new collagenor gelatin-coated plates in alpha-MEM expansion medium containing $1 \%$ penicillin/streptomycin, 10\% FBS, NEAA, and L-glutamine. The expansion medium was replenished every 3-4 days. iMSC like cells cultured on gelatin (iMSCs/G) or on collagen (iMSCs/C) were passaged when they reached $70 \%$ subconfluency at a ratio of $1: 3$ as completely single cell suspensions. Derivation iMSCs P4 to P7 times were used for all experiments. Derivation experiment of every iPSC clones was performed at five times.

2.4. RNA Preparation and Semiquantitative Reverse Transcription Polymerase Chain Reaction (RT-PCR). RNA was 
(a)

iPS cells onto MEF

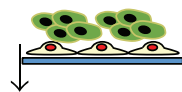

Feeder free culturing

(1)

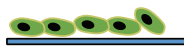

Gelatin

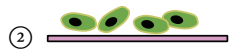

$\downarrow$ Type I collagen (b)

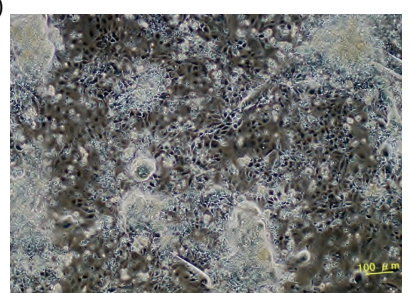

(c)

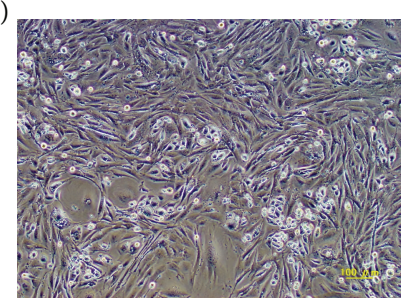

$\begin{array}{lc}\text { Dissociate } & \text { (medium change every } 3-4 \text { days) } \\ \text { and replate } & \text { Passage at } 1: 3 \text { split ratio } \\ \text { (Passage } 0) & \end{array}$
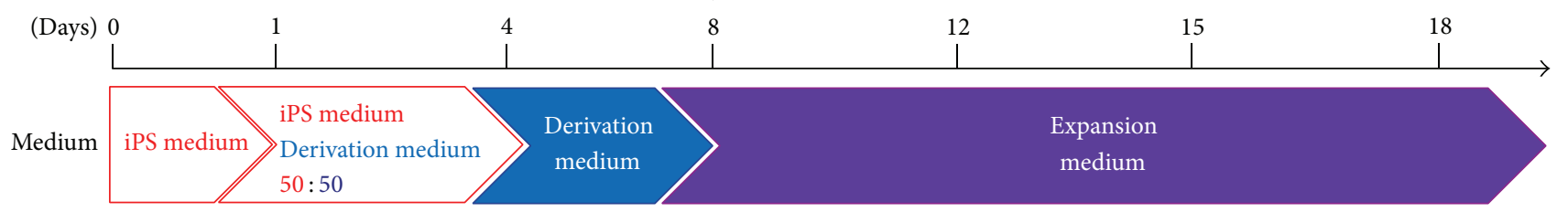

Factors LIF

Dexamethasone

Ascorbic acid

(d)
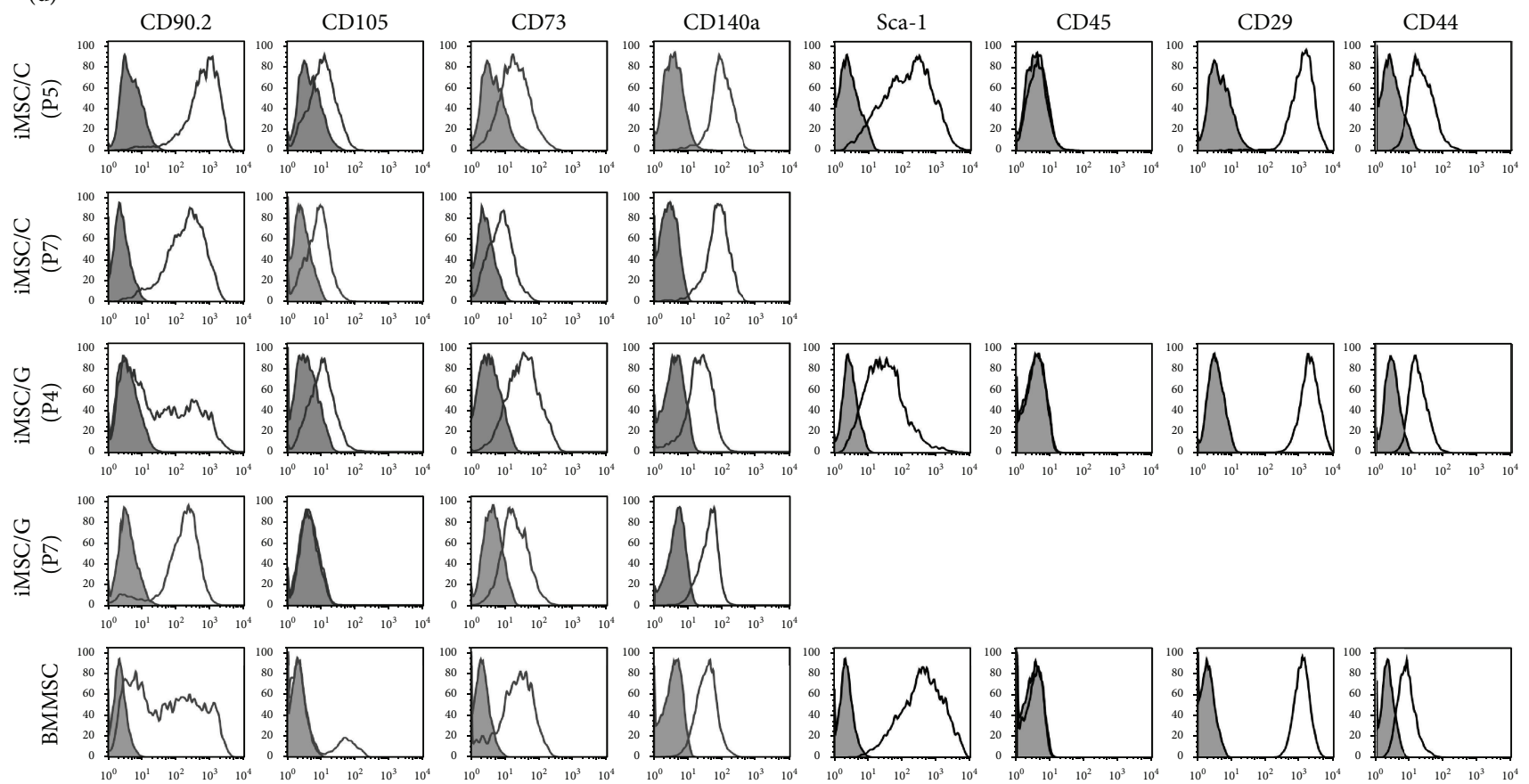

FIgURE 1: Derivation of mouse iPSC-derived mesenchymal stem cells (iMSC). (a) Protocol for derivation of iMSCs. iPSCs were maintained on mouse embryonic fibroblast (MEF) in iPS medium with leukemia inhibitory factor (LIF) and harvested and dissociated into single cells that were seeded into gelatin (1)- or collagen (2)-coated plates. After 1 day, the iPS medium was supplemented with an equal volume of the derivation medium with dexamethasone and ascorbic acid, and after 2 days, the medium was replaced with derivation medium. After 8 days, cells (passage zero; P0) were harvested and replaced in new gelatin- or collagen-coated plates in expansion medium. (b) Adherent spindleshaped fibroblast-like cells at P0 appeared on 8-derivation day. (c) Homogenous fibroblast-like cells at P3. (d) Characterization of iMSCs on gelatin (iMSCs/G) and on collagen (iMSCs/C) at P4 to P7. Bone marrow (BM) MSC at P5 was provided by cell sorting from mouse bone marrow. The gray histogram represents the negative control staining with fluorescence-conjugated isotype IgG and white overlay represents antigen at CD90.2, CD105, CD73, CD29, CD44, CD45, Sca-1, and CD140 $\alpha$. 
extracted from P5 iPSCs, BMMSCs, iMSCs/G, iMSCs/C, and mouse MSCs (Invitrogen, Carlsbad, CA, USA) using an RNeasy Mini Kit (Qiagen, Valencia, CA, USA) according to the manufacturer's instructions with on-column DNase I digestion. Mouse MSCs were cultured in DMEM supplemented with $10 \%$ FBS and antibiotics under $5 \% \mathrm{CO}_{2}$ at $37^{\circ} \mathrm{C}$. When the cells reached $\sim 70 \%$ confluence, they were passaged by using $0.25 \%$ trypsin/EDTA. Expanded MSCs at P4 to P7 times were used for all experiments. RT was carried out using the PrimeScript II First Strand cDNA Synthesis Kit (Takara Bio, Otsu, Japan). PCR amplification was performed in a PCR Thermal Cycler (Dice, Takara) using an Ex Taq (Takara) (Catalog \#6210A), with the standard cycle condition. Germlayer associated primers Nanog, Oct4, and SOX2 for undifferentiated cells; Nestin, Otx2, TP63/TP73L, SOX-2, and SOX1 for ectodermal lineage; AFP, GATA-4, PDX-1/IPF1, SOX17, and $H N F-3 b / F o x A 2$ for endodermal lineage; Brachury for mesodermal lineage were purchased from Mouse Pluripotent Stem Cell Assessment Primer Pair Panel Kit (R\&D systems) (Catalog \#SC015), and other primers (PDGFR- $\alpha$, PDGFR- $\beta$, $A C T I N$, and SOX1) were prepared as previously described (Supplementary Table 1 in Supplementary Material available online at http://dx.doi.org/10.1155/2016/9013089) [20]. Total RNA (500 ng) was used as a template for cDNA synthesis with the RT ${ }^{2}$ First Strand (Qiagen) kit (Catalog \#330401). The product was assessed by $1.5 \%$ agarose gel electrophoresis with ethidium bromide staining.

2.5. Microarray Analysis. P5 iPSCs, BMMSCs (P5), iMSCs/G (5-6P), and iMSCs/C (5-6P) were analyzed with a mouse mesenchymal stem cell $\mathrm{RT}^{2}$ Profiler PCR Array (Qiagen) (Catalog \#PAMM-082Z). Real-time PCR was carried out on an ABI Prism 7500 system format C using a RT $^{2}$ SYBR Green ROX $^{\mathrm{TM}}$ qPCR Mastermix (Qiagen) (Catalog \#330522) under standard cycling conditions. Data were analyzed using $\Delta \Delta \mathrm{C}_{\mathrm{t}}$ method for 96-well format. The value of each sample was normalized to the expression level of the GAPDH housekeeping gene in the same sample. Relative mRNA expression level was expressed as fold-increases of the target genes to GAPDH mRNA level. A clustergram was generated by hierarchical clustering of genes and samples were displayed in a heat map, with dendrograms indicating coregulated genes across groups or individual samples. A scatter plot was used to compare the normalized expression of every gene in the array between the two selected groups by plotting them against one another. Every cell lines composed of three samples were analyzed at three times.

2.6. Flow Cytometric Analysis. Surface markers for mouse MSCs were quantified by flow cytometry using antibodies against CD11b, CD29 (encoded by Itgb1 gene), CD44, CD73, CD90.2 (Thy1), CD105 (Eng) (from eBiosciences, San Diego, CA, USA), CD31, CD34, CD45, Sca-1, and PDGFR- $\alpha$ (Biolegend, San Diego, CA, USA) antibodies. Nonspecific fluorescence was determined with isotype-matched antibodies (BD Biosciences, Franklin Lakes, NJ, USA) (Supplementary Table 2). BMMSCs, iMSCs/G, iMSCs/C, and iPSCs $(1 \times$ $10^{5}$ cells) were collected by trypsinization and washed once with DMEM supplemented with 5\% FBS. The sample was resuspended in $100 \mu \mathrm{L}$ of $5 \% \mathrm{FBS} / \mathrm{PBS}$ containing each of monoclonal antibodies and then incubated for $30 \mathrm{~min}$ at $4^{\circ} \mathrm{C}$. The nonspecific binding of APC, FITC, and PE conjugates was determined using isotype antibodies (all from eBiosciences). The cells were then washed twice and resuspended in $400 \mu \mathrm{L}$ of $5 \%$ FBS/PBS. Cells were sorted on a FACSCalibur flow cytometer (BD Biosciences) and results were analyzed with Flow Jo software (Tree Star, Ashland, OR, USA). Flow cytometry analyses were repeated at least three times.

2.7. Differentiation Assay. We evaluated the potential of P5 iMSCs/G or iMSCs/C derived from each of iPSC lines to differentiate into adipocytes, osteocytes, and chondrocytes. These differentiations were performed using a human MSC differentiation kit (Lonza, Walkersville, MD, USA). Adipose differentiation was performed on normal coated plates and osteoblast differentiation on collagen-coated plates. Positive areas in Alizarin red for osteogenesis and Oil Red-O dye for adipogenesis were examined under higher magnification (4 or 10x) and quantitated using Image J. The positive density was expressed as the mean positive area per high-power field. A total of 4 high-power fields were randomly examined and counted from each treatment group ( $n=3$ /group). The statistical significance of differences was determined using Student's $t$-test for two group comparisons or oneway analysis of variance for multiple comparisons, followed by Tukey's test. Differences with a value of $P<0.05$ were considered significant. Differentiation assay for iMSC lines was repeated at three times.

\section{Results}

3.1. Derivation of Mouse iMSCs from iPSCs. To obtain the mouse MSC-like cells, we employed the modified version of the one-step derivation method used to obtain human MSClike cells from iPSCs [3] (Figure 1(a)). With this method, adherent spindle-shaped fibroblast-like cells appeared in the derivation medium on 8 -derivation day that continued to proliferate in the expansion medium (Figure 1(b)). After more than three passages, homogenous fibroblast-like derivation cells were obtained on both gelatin- and collagen-coated plates, which exhibited similar numbers of cells and doubling times (Figure 1(c)). Removal of MEF cells from iPSCs was completed using microbeads (Supplementary Figure 1).

\subsection{Characterization of Surface Antigens in Fibroblast-Like} Adherent Cells Derived from Mouse iPSCs. To characterize adherent fibroblast-like derivation cells, the expression of putative MSC markers was assessed at several time points. About $95 \%$ of derivation cells on collagen- or gelatin-coated plates were positive for putative MSC markers including Sca1, CD29 (Itgb1), CD44, and CD73, but negative for CD11b, CD31, CD45, and CD117, independent of passage number (Figure 1(d)). In contrast, derivation cells heterogeneously expressed CD90 (Thy1) and PDGFR- $\alpha$ at P0 but gradually upregulated their markers dependent on passage numbers (Figure 1(d)). Expression of CD105 (Eng) was downregulated 


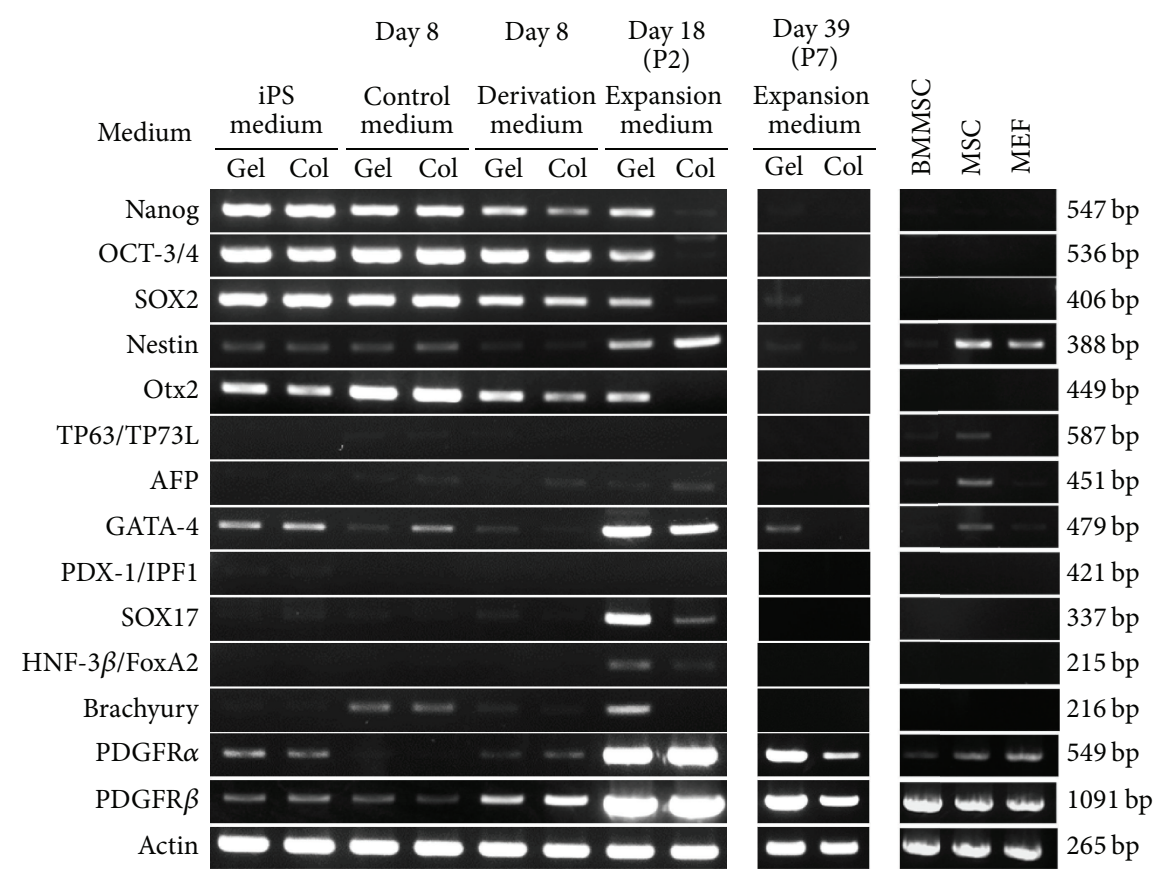

FIGURE 2: Effects of gelatin or collagen on germ-layer associated genes expression in iMSCs. Time-course (day 8 to day 39 ) genes expression in each cell with semiquantitative reverse transcription polymerase chain reaction. iPS medium; iPSCs on gelatin (Gel) or collagen (Col), control medium; iPSCs in 8 day-derivation without dexamethasone and ascorbic acid, derivation medium; iPSCs in 8 day-derivation, expansion medium; iMSCs at passages 2 (P2) and P7 in expansion medium, BMMSCs; bone marrow MSC at P5, MSC; MSCs from Invitrogen, and mouse embryonic fibroblast (MEF).

with increasing numbers of passages in derivation cells on gelatin, but not on collagen (Figure $1(\mathrm{~d})$ ). This heterogeneous expression of CD90 and CD105 was also detected in BMMSCs at P1 (Figure 1(d)). Our prospectively isolated BMMSCs using FACS analysis were negative for CD45. Collectively, the expression profiles in derivation cells on gelatin- or collagencoated plates were similar to the general MSC profile [21]. Previous studies showed that immunophenotypic profiles of MSCs vary by species, cell source, and passage number [2224].

3.3. Effects of Gelatin or Collagen on Germ-Layer Associated Genes Expression in iMSCs. Stemness genes including Nanog, Oct4, and Sox 2 were expressed in iPSCs. Following derivation, their expression was gradually reduced and finally disappeared in iMSCs/C, although Oct4 and Sox2 transcripts persisted in iMSCs/G until 18-derivation day (Figure 2). Expression of Otx2 as an ectodermal lineage marker was also detected in iMSCs/G, but not iMSCs/C after derivation. Nestin (Nes) and GATA-4 levels were upregulated in both iMSCs after derivation, while SOX17 was markedly upregulated in iMSCs/G as compared to iMSCs/C. Brachyury, PDGFR- $\alpha$, and PDGFR- $\beta$ as a mesodermal lineage gene were upregulated in iMSCs/C and iMSCs/G following derivation. BMMSCs expressed PDGFR- $\alpha$ and PDGFR- $\beta$ but had a little Nestin, TP63/TP73L, and AFP expression. These findings suggest that germ-layer associated gene expression profiles are similar in iMSCs/G and C, although stemness associated gene expression tended to persist.
3.4. Mesenchymal Stem Cell Associated Gene Expression in Mouse iPSCs, BMMSCs, iMSCs/G, and iMSCs/C. To investigate whether there is a molecular analogy among 4 cell groups as iPSCs, BMMSCs (P5), iMSCs/G (P5), and iMSCs/C (P5), we compared their gene expression profiles using a PCR array (Supplementary Figure 2). We performed a clustering analysis by selecting 50 genes that were upregulated by 2fold in BMMSCs relative to iPSCs (Supplementary Figure 3). The 50 genes clustering analysis divided the cells into three clusters, iPSCs, BMMSCs, and iMSCs/G/C with a stronger correlation between iMSCs/G/C and BMMSCs as opposed to iPSCs (Figure 3(a)). We further examined 19 of 50 MSCspecific upregulated genes and found that BMMSCs and iMSCs/G and iMSCs/C were correlated by MSC-specific gene clustering (Figure 3(b)). A subclustering analysis based on six stemness genes upregulated Lif in iMSCs/G, but not in iMSCs/C. We added pairwise analyses ( $>2$-fold), excluding genes with low expression selected by combining cell groups. The pairwise analysis between iMSCs/G and iMSCs/C revealed upregulation of Sox2, Bmp2, and Lif but downregulation of Tbx5, PPAR $\gamma$, and Sox 9 in iMSCs/G (Figure 3(c)). The scatter plots of twofold upregulated MSCspecific gene expressions (14 genes) demonstrated a resemble pattern (stars) between BMMSCs and iMSCs, except for common upregulation of Bmp2, Mcam (CD146), and Alcam (CD166) in iMSCs (Figures 3(d1) and 3(d2)). Alcam encodes CD166, which is a member of the immunoglobulin superfamily proteins. Mcam functions as a receptor for laminin $\alpha 4$, a matrix molecule. MSC-specific genes were 


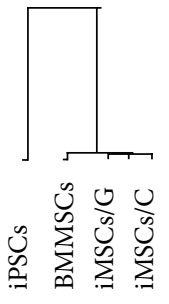

(a)
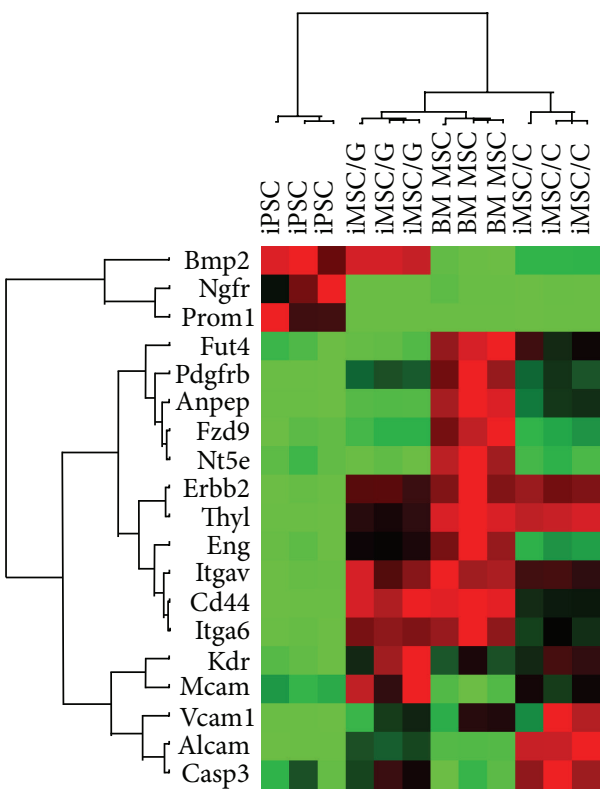

(b)

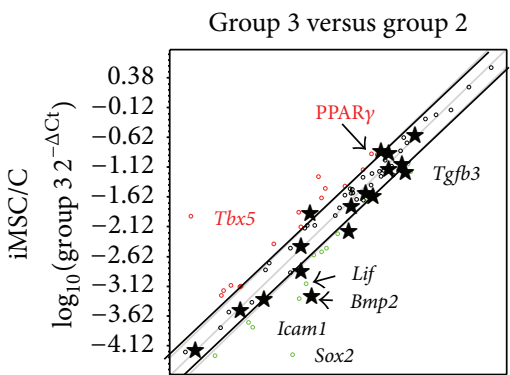

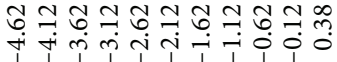

$\log _{10}\left(\right.$ group $\left.22^{-\Delta C t}\right)$

iMSC/G

(c)

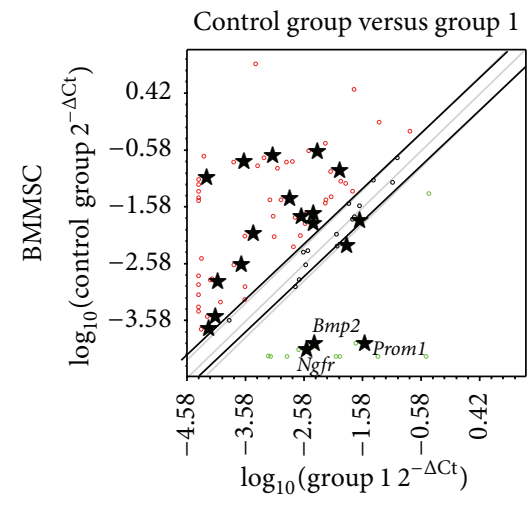

iPSC

(e1)

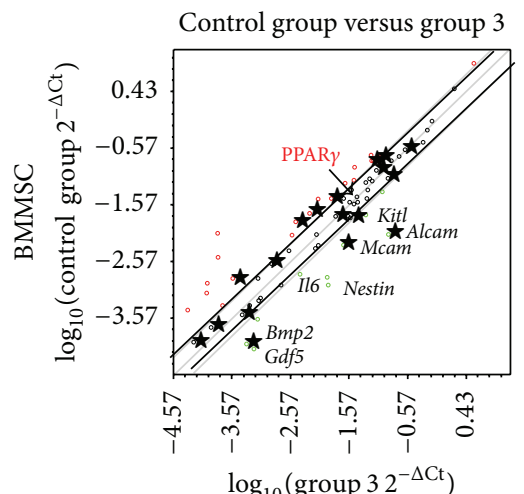

iMSC/C

(d1)

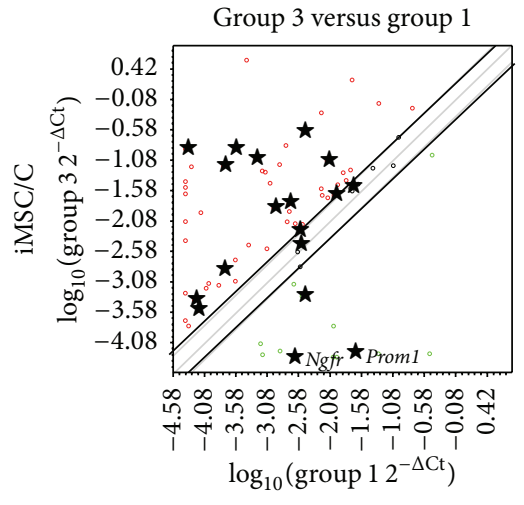

iPSC
Control group versus group 2

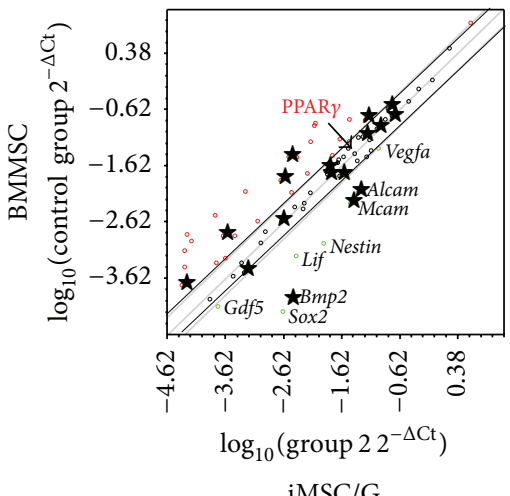

(d2)

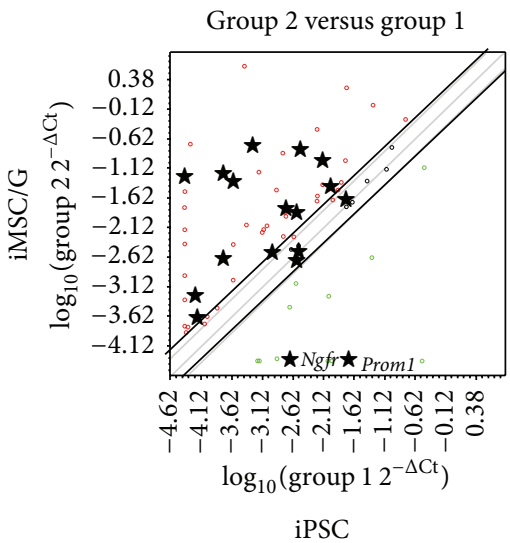

(e3)

Figure 3: Microarray analysis of iMSCs/G, iMSCs/C, BMMSCs, and iPSCs. (a) Hierarchical cluster analysis of iMSCs/G, iMSCs/C, BMMSCs, and iPSCs by selecting 50 genes with upregulation by 2-fold in BMMSCs relative to iPSCs. (b) Hierarchical clustering analysis of MSC-specific genes of iMSCs/G, iMSCs/C, BMMSCs, and iPSCs. (c-e3) Pairwise scatter plots comparisons of the gene expression profiles of iMSC/C versus $\mathrm{iMSC} / \mathrm{G}$ (c), BMMSC versus iMSC/C (d1)/iMSC/G (d2), BMMSC versus iPSC (e1), iMSC/C/iPSC (e2), and iMSC/G/iPSC (e3). Diagonal lines indicate the boundaries of twofold changes in gene expression. Gene expression levels are shown on $\log _{10}$ scale. MSC-specific genes: stars $(\star)$. 
generally upregulated in BMMSCs, iMSCs/G, and iMSCs/C compared with iPSCs except for $\mathrm{Ngfr}$ and Prom1 (CD133) according to each pairwise analysis (Figures 3(e1)-3(e3)). $N g f r$ is a member of the tumor necrosis factor receptor superfamily, and Prom1 encodes CD133 which is a member of pentaspan transmembrane glycoproteins [25]. Collectively, these results indicate that this one-step derivation method induced molecular similarities in terms of MSC-specific gene expression between BMMSCs and iMSCs/G or iMSCs/C.

\subsection{Derivation of iMSCs on Gelatin-Coated Plates Enhances} Osteoblast Differentiation Potential. We assessed the osteogenic differentiation property of iMSCs/G and iMSCs/C. iMSCs were maintained at $70 \%-80 \%$ confluency on gelatinor collagen-coated plates in osteogenic differentiation medium for 28 days, with medium replacement every 3-4 days. The majority of iMSCs/G demonstrated positive staining with Alizarin red and much amounts of its positive deposits, whereas a small fraction of iMSCs/C showed positive staining with a few amounts of deposits with quantification analysis (Figures 4(a) and 4(b)) (Supplementary Figure 4). There were no Alizarin red-positive cells on either substrate without induction of osteogenic differentiation. These findings at 28osteoblast induction day suggested that iMSCs/G differentiate into osteoblasts more efficiently than iMSCs/C, which was further supported by the upregulation of Bmp2 (6.50fold) and Lif (4.77-fold) in iMSCs/G in pairwise scatter plots, prior to osteogenic induction. We performed the induction of chondrocyte differentiation of iMSCs by micromass culture method. The number of Alcian Blue positive cells was similar in both iMSCs on 24-differentiation day (Figure 4(c)), and the clustergram of chondrogenesis-associated gene expressions revealed molecular similarity between iMSCs/G and iMSCs/C.

3.6. Derivation of iMSCs on Collagen-Coated Plates Promoted Adipose Differentiation. Adipogenic differentiation of both iMSCs was examined on 7- and 21-differentiation day. The number of Oil Red-O-positive droplets was much higher in iMSCs/C as compared to iMSCs/G (Figures 4(d) and 4(e)) (Supplementary Figure 4). This indicates the propensity for adipogenesis in iMSCs/C. PPAR $\gamma$ was upregulated by 2.18 fold in iMSCs/C according to pairwise analysis, prior to adipose induction.

\section{Discussion}

In this study, differentiation assay using uncoated plates revealed that derived mouse iPS-iMSCs on gelatin and collagen coating efficiently differentiated into osteoblast and adipose lineages, respectively. A previous study showed that rat BMMSCs showed increased proliferation and differentiation into a neurogenic lineage when grown on gelatin-coated plates as compared to noncoated plates, whereas adipose and osteogenic differentiation potentials were unaffected by gelatin [10]. In other studies using human or mouse derived iMSCs, osteogenic or chondrogenic differentiation potential was induced by gelatin or collagen coating, while they did not directly compare iMSC differentiation potential by deriving on the two types of substrates $[3,12,15,26]$. The present study investigated for the first time how gelation or collagen coating affects the differentiation potential of derived iMSCs in culture.

In our pairwise scatter plot analysis, iMSCs/G upregulated Bmp2 and Lif prior to induction of osteogenic or adipose differentiation, whereas iMSCs/C upregulated PPAR $\gamma$. MSCs can differentiate into bone and adipose tissue under the control of Lif and PPAR $\gamma$ [27]. Lif is a member of the interleukin- 6 family of gp130 cytokines which stimulates osteoblast differentiation and reduces adipogenesis in bone marrow stromal cells $[28,29]$. PPAR $\gamma$ acts as a dominant negative regulator of osteoblast differentiation in BMMSCs [30] and inhibits Lif-induced proliferation of mouse embryonic stem cells [31]. However, the mutual interaction between Lif and PPAR $\gamma$ in iMSCs has not been addressed and elucidated. Our present results suggest that, during iMSCs derivation from iPSCs, extracellular matrix composition biases its differentiation potential towards adipose or osteoblast lineages via up- or downregulation of these master genes. Gelatin which is composed of collagen-peptides can form three-dimensional complex structures, possibly inducing differences in germlayer associated potentials of iPSCs. This hypothesis is compatible with previous reports [32-34]. However, threedimensional relevance of gelatin remains unresolved in this study.

The cluster analysis of MSC-specific genes demonstrated that iMSCs/G or iMSCs/C were more closely related to BMMSCs than to iPSCs. This finding was confirmed by the pairwise scatter plot analysis which revealed a molecular similarity between BMMSCs and iMSCs/G or iMSCs/C, although they differed in terms of expression of Alcam and Mcam involved in cell adhesion molecules. We cultured BMMSCs on noncoated plates, which may explain differences in gene expressions between BMMSCs and iMSCs. A recent study showed that the gene expression profile of human iMSCs grown on gelatin-coated plates was similar to that of human BMMSCs by hierarchical cluster and MSC-specific gene heat-map analyses [12]. Nonetheless, our findings indicate that one-step iMSC derivation method can generate mouse iMSCs that have molecular characteristics compatible with those of primary mouse BMMSCs. The pairwise scatter plots demonstrated that the patterns of up- and/or downregulation of MSC-specific genes were similar between iPSCs and iMSCs as well as BMMSCs, suggesting the possibility that the process of in vitro iMSC derivation from iPSCs may reflect the generation process of BMMSCs in vivo.

As expected, we found that the expression of stemness related genes was gradually downregulated during the derivation of iMSCs from iPSCs. In addition, the expression stemness and germ-layer-specific genes persisted in iMSCs/G as compared to iMSCs/C. In contrast, germ-layer associated genes such as Nestin, GATA4, SOX17, Brachury, PDGFR $\alpha$, and PDGFR $\beta$ were transiently upregulated in iMSCs at P2, but these genes were downregulated with additional passages, with the exception of GATA4, PDGFR $\alpha$, and PDGFR $\beta$. This is consistent with the gene expression profiles of human iMSCs $[12,13]$. 


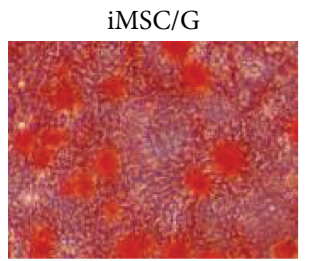

iMSC/G

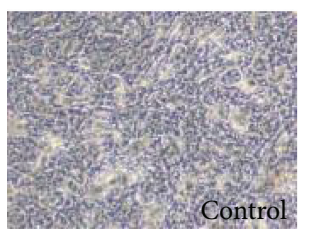

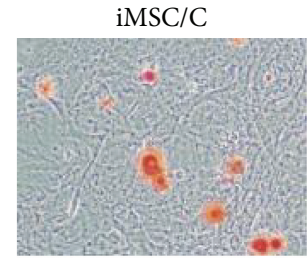

$\mathrm{iMSC} / \mathrm{C}$

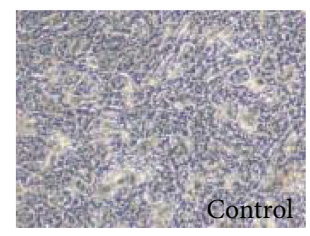

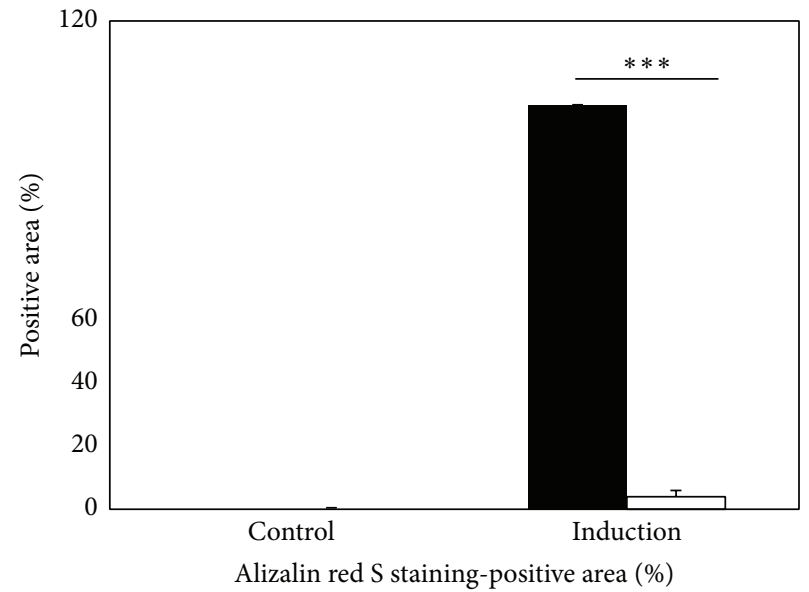

Alizalin red S staining-positive area (\%)

iMSC/G

$\square \mathrm{iMSC} / \mathrm{C}$

(a)

iMSC/G

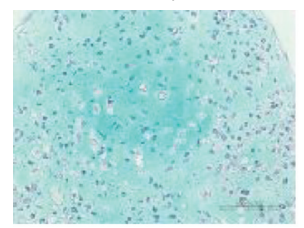

iMSC/G

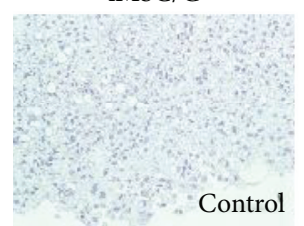

$\mathrm{iMSC} / \mathrm{C}$

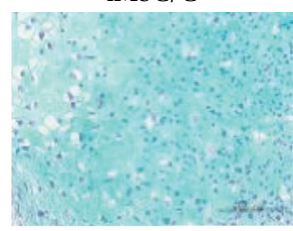

$\mathrm{iMSC} / \mathrm{C}$

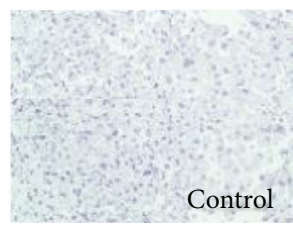

(c)

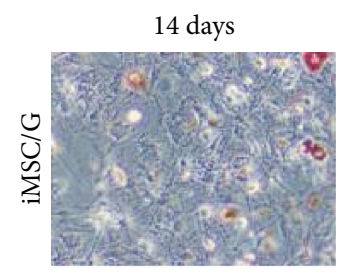

14 days

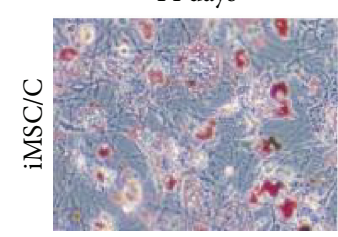

(b)
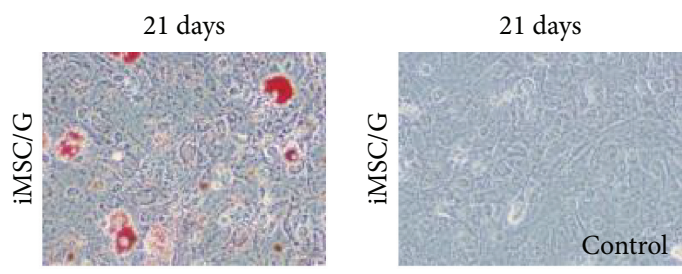

21 days

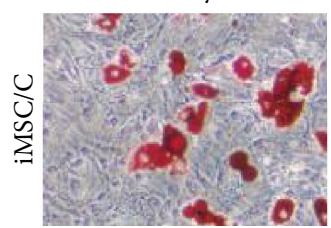

(d)

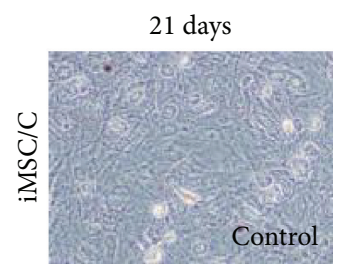

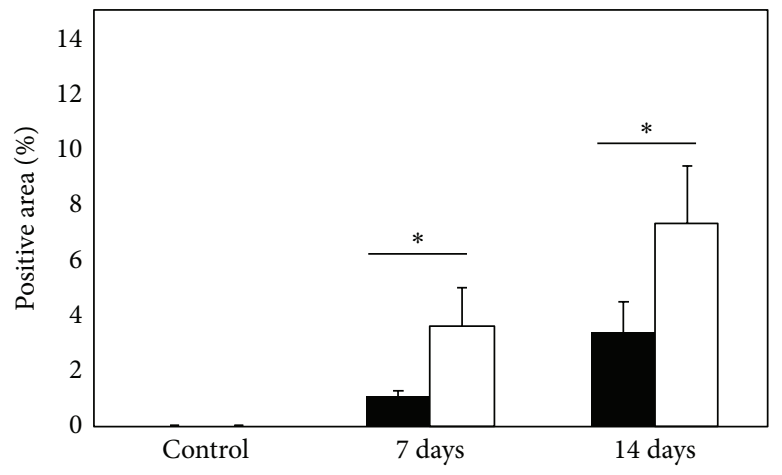

iMSC/G

$\square \mathrm{iMSC} / \mathrm{C}$

(e)

FIGURE 4: Osteogenic and adipose differentiation of iMSC/G and C derived from an iPSC clone (2A-4F-60). (a) iMSCs cultured in medium containing osteogenic differentiation factors (induction) or absence (control) and stained with Alizarin red at 28 days. (b) Relative amount of Alizarin red-positive areas. ${ }^{* * *} p$ value $<0.001$. (c) Chondrocyte differentiation of iMSCs by micromass culture. (d) iMSCs cultured in differentiation medium (induction) or absence (control) and stained Oil Red-O at 14 and 21 days. (e) Relative amount of Oil Red-O-positive areas. ${ }^{*} p$ value $<0.05$. 
Previous studies have reported that expression levels of $\mathrm{CD} 105$ as an affinity coreceptor for transforming growth factor- (TGF-) $\beta 1$ and TGF- $\beta 3$ gene are correlated with MSC properties and are changed during in vitro expansion [14, 24, $35,36]$. In the present study, iMSCs/G gradually decreased CD105 expression over many passages and differentiated into osteoblasts more efficiently than iMSCs/C. This finding is in accordance with a previous study demonstrating that CD105 depletion enhanced human adipose-derived stromal cell osteogenesis via reduction of TGF- $\beta 1$ [24]. In this study, we observed upregulation of TGF- $\beta 3$ (3.01 fold), but not TGF$\beta 1$ in iMSC/G.

\section{Conclusion}

We demonstrated the molecular properties of mouse iMSCs/G or iMSCs/C derived from iPSCs by a simple one-step derivation method. Deriving on gelatin enhanced the osteogenic differentiation potential of derived iMSCs via upregulation of Lif and Bmp2, whereas their adipogenesis was enhanced via upregulation of PPAR $\gamma$ by deriving on collagen. These findings indicate that extracellular matrix components such as gelatin and collagen are critical regulators of the iMSC differentiation potential. The mouse iMSCs established by this method can be used as a tool for precisely analyzing anti-inflammatory or immunoregulatory roles of MSCs in a congenic mouse models, in addition to serving as an alternative cell source of primary MSCs and an off-the-shelf product.

\section{Competing Interests}

The authors declare that there are no competing interests.

\section{Authors' Contributions}

Chizuka Obara, Kazuya Takizawa, and Katsushi Tajima contributed equally to this study.

\section{Acknowledgments}

The authors thank Tomoko Fukuzaki, Katsuko Noshiro, and Michio Hama for their technical support. This work was supported by a grant from Kanehara and also by grants-inaid for scientific research KAKENHI Grants nos. 25861143 (M. Hazawa) and 80292423 (K. Tajima) from the Ministry of Education, Culture, Sports, Science, and Technology of Japan.

\section{References}

[1] H. D. Zomer, A. S. Vidane, N. N. Goncalves, and C. E. Ambrosio, "Mesenchymal and induced pluripotent stem cells: general insights and clinical perspectives," Stem Cells and Cloning: Advances and Applications, vol. 8, pp. 125-134, 2015.

[2] R. A. Webster, S. P. Blaber, B. R. Herbert, M. R. Wilkins, and G. Vesey, "The role of mesenchymal stem cells in veterinary therapeutics-a review," New Zealand Veterinary Journal, vol. 60 , no. 5 , pp. $265-272,2012$.
[3] Y. Liu, A. J. Goldberg, J. E. Dennis, G. A. Gronowicz, and L. T. Kuhn, "One-step derivation of mesenchymal stem cell (MSC)like cells from human pluripotent stem cells on a fibrillar collagen coating," PLoS ONE, vol. 7, no. 3, Article ID e33225, 2012.

[4] D. Dey and G. R. D. Evans, "Generation of induced pluripotent stem (iPS) cells by nuclear reprogramming," Stem Cells International, vol. 2011, Article ID 619583, 11 pages, 2011.

[5] M. Giuliani, N. Oudrhiri, Z. M. Noman et al., "Human mesenchymal stem cells derived from induced pluripotent stem cells down-regulate NK-cell cytolytic machinery," Blood, vol. 118, no. 12, pp. 3254-3262, 2011.

[6] Y. Jung, G. Bauer, and J. A. Nolta, "Concise review: induced pluripotent stem cell-derived mesenchymal stem cells: progress toward safe clinical products," STEM CELLS, vol. 30, no. 1, pp. 42-47, 2012.

[7] O. Lindvall and Z. Kokaia, "Stem cells in human neurodegenerative disorders-time for clinical translation?" The Journal of Clinical Investigation, vol. 120, no. 1, pp. 29-40, 2010.

[8] F. Guilak, D. M. Cohen, B. T. Estes, J. M. Gimble, W. Liedtke, and C. S. Chen, "Control of stem cell fate by physical interactions with the extracellular matrix," Cell Stem Cell, vol. 5, no. 1, pp. 17-26, 2009.

[9] B. Trappmann, J. E. Gautrot, J. T. Connelly et al., "Extracellularmatrix tethering regulates stem-cell fate," Nature Materials, vol. 11, pp. 642-649, 2012.

[10] Y. H. Park, J. I. Yun, N. R. Han et al., "Mass production of early-stage bone-marrow-derived mesenchymal stem cells of rat using gelatin-coated matrix," BioMed Research International, vol. 2013, Article ID 347618, 10 pages, 2013.

[11] S. B. Lee, D. Seo, D. Choi et al., "Contribution of hepatic lineage stage-specific donor memory to the differential potential of induced mouse pluripotent stem cells," STEM CELLS, vol. 30, no. 5, pp. 997-1007, 2012.

[12] J. Frobel, H. Hemeda, M. Lenz et al., "Epigenetic rejuvenation of mesenchymal stromal cells derived from induced pluripotent stem cells," Stem Cell Reports, vol. 3, no. 3, pp. 414-422, 2014.

[13] Y. S. Chen, R. A. Pelekanos, R. L. Ellis, R. Horne, E. J. Wolvetang, and N. M. Fisk, "Small molecule mesengenic induction of human induced pluripotent stem cells to generate mesenchymal stem/stromal cells," Stem Cells Translational Medicine, vol. 1, no. 2, pp. 83-95, 2012.

[14] F. A. A. Ishiy, R. D. Fanganiello, K. Griesi-Oliveira et al., "Improvement of in vitro osteogenic potential through differentiation of induced pluripotent stem cells from human exfoliated dental tissue towards mesenchymal-like stem cells," Stem Cells International, vol. 2015, Article ID 249098, 9 pages, 2015.

[15] Y. Dogaki, S. Y. Lee, T. Niikura et al., "Efficient derivation of osteoprogenitor cells from induced pluripotent stem cells for bone regeneration," International Orthopaedics, vol. 38, no. 9, pp. 1779-1785, 2014.

[16] H. Sakurai, Y. Sakaguchi, E. Shoji et al., "In vitro modeling of paraxial mesodermal progenitors derived from induced pluripotent stem cells," PLoS ONE, vol. 7, no. 10, Article ID e47078, 2012.

[17] R. Araki, M. Uda, Y. Hoki et al., "Negligible immunogenicity of terminally differentiated cells derived from induced pluripotent or embryonic stem cells," Nature, vol. 494, no. 7435, pp. 100-104, 2013.

[18] S. Morikawa, Y. Mabuchi, Y. Kubota et al., "Prospective identification, isolation, and systemic transplantation of multipotent 
mesenchymal stem cells in murine bone marrow," The Journal of Experimental Medicine, vol. 206, no. 11, pp. 2483-2496, 2009.

[19] C. Obara, K. Tomiyama, K. Takizawa et al., "Characteristics of three-dimensional prospectively isolated mouse bone marrow mesenchymal stem/stromal cell aggregates on nanoculture plates," Cell and Tissue Research, 2016.

[20] Y. Takashima, T. Era, K. Nakao et al., "Neuroepithelial cells supply an initial transient wave of MSC differentiation," Cell, vol. 129, no. 7, pp. 1377-1388, 2007.

[21] M. Dominici, K. Le Blanc, I. Mueller et al., "Minimal criteria for defining multipotent mesenchymal stromal cells. The International Society for Cellular Therapy position statement," Cytotherapy, vol. 8, no. 4, pp. 315-317, 2006.

[22] P. C. Sachs, M. P. Francis, M. Zhao et al., "Defining essential stem cell characteristics in adipose-derived stromal cells extracted from distinct anatomical sites," Cell and Tissue Research, vol. 349, no. 2, pp. 505-515, 2012.

[23] X.-F. Yang, X. He, J. He et al., "High efficient isolation and systematic identification of human adipose-derived mesenchymal stem cells," Journal of Biomedical Science, vol. 18, no. 1, article 59, 2011.

[24] B. Levi, D. C. Wan, J. P. Glotzbach et al., "CD105 protein depletion enhances human adipose-derived stromal cell osteogenesis through reduction of transforming growth factor $\beta 1$ (TGF- $\beta 1$ ) signaling," The Journal of Biological Chemistry, vol. 286, no. 45, pp. 39497-39509, 2011.

[25] D. Johnson, A. Lanahan, C. R. Buck et al., "Expression and structure of the human NGF receptor," Cell, vol. 47, no. 4, pp. 545-554, 1986.

[26] T. Teramura, Y. Onodera, T. Mihara, Y. Hosoi, C. Hamanishi, and K. Fukuda, "Induction of mesenchymal progenitor cells with chondrogenic property from mouse-induced pluripotent stem cells," Cellular Reprogramming, vol. 12, no. 3, pp. 249-261, 2010.

[27] C. Nombela-Arrieta, J. Ritz, and L. E. Silberstein, "The elusive nature and function of mesenchymal stem cells," Nature Reviews Molecular Cell Biology, vol. 12, no. 2, pp. 126-131, 2011.

[28] J. Aubert, S. Dessolin, N. Belmonte et al., "Leukemia inhibitory factor and its receptor promote adipocyte differentiation via the mitogen-activated protein kinase cascade," The Journal of Biological Chemistry, vol. 274, no. 35, pp. 24965-24972, 1999.

[29] U. A. White and J. M. Stephens, "The gp130 receptor cytokine family: regulators of adipocyte development and function," Current Pharmaceutical Design, vol. 17, no. 4, pp. 340-346, 2011.

[30] M. J. Jeon, J. A. Kim, S. H. Kwon et al., "Activation of peroxisome proliferator-activated receptor- $\gamma$ inhibits the Runx2-mediated transcription of osteocalcin in osteoblasts," The Journal of Biological Chemistry, vol. 278, no. 26, pp. 23270-23277, 2003.

[31] C. Mo, W. Chearwae, and J. J. Bright, "PPAR $\gamma$ regulates LIFinduced growth and self-renewal of mouse ES cells through Tyk2-Stat3 pathway," Cellular Signalling, vol. 22, no. 3, pp. 495500, 2010.

[32] F. Faghihi, E. Mirzaei, J. Ai et al., "Differentiation potential of human chorion-derived mesenchymal stem cells into motor neuron-like cells in two- and three-dimensional culture systems," Molecular Neurobiology, vol. 53, no. 3, pp. 1862-1872, 2016.

[33] X. Liu and P. X. Ma, "Phase separation, pore structure, and properties of nanofibrous gelatin scaffolds," Biomaterials, vol. 30, no. 25, pp. 4094-4103, 2009.
[34] B. Chevallay, N. Abdul-Malak, and D. Herbage, "Mouse fibroblasts in long-term culture within collagen three-dimensional scaffolds: influence of crosslinking with diphenylphosphorylazide on matrix reorganization, growth, and biosynthetic and proteolytic activities," Journal of Biomedical Materials Research, vol. 49, no. 4, pp. 448-459, 2000.

[35] F. Li, S. Bronson, and C. Niyibizi, "Derivation of murine induced pluripotent stem cells (iPS) and assessment of their differentiation toward osteogenic lineage," Journal of Cellular Biochemistry, vol. 109, no. 4, pp. 643-652, 2010.

[36] P. Anderson, A. B. Carrillo-Gálvez, A. García-Pérez, M. Cobo, and F. Martín, "CD105 (Endoglin)-negative murine mesenchymal stromal cells define a new multipotent subpopulation with distinct differentiation and immunomodulatory capacities," PLoS ONE, vol. 8, no. 10, Article ID e76979, 2013. 

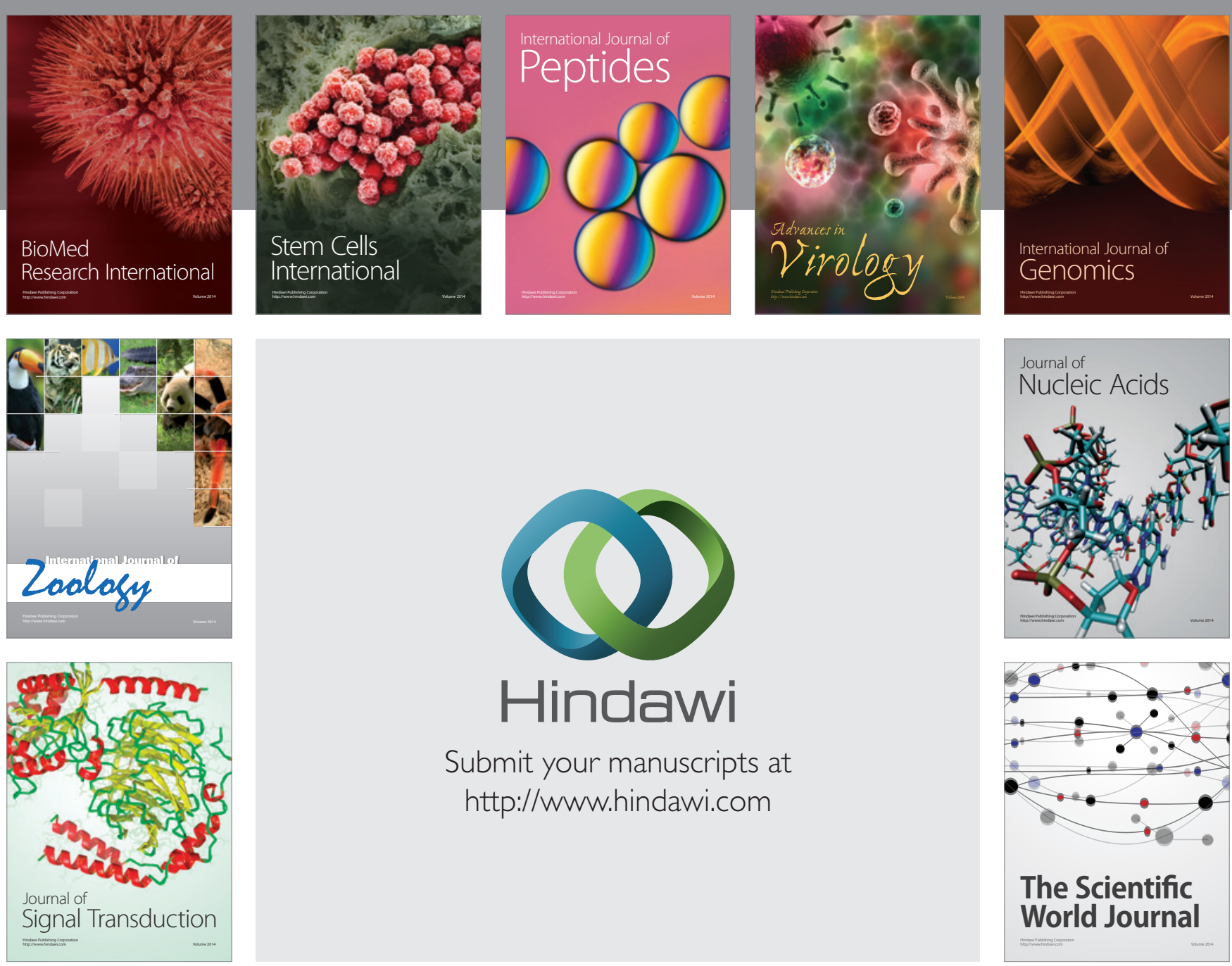

Submit your manuscripts at

http://www.hindawi.com
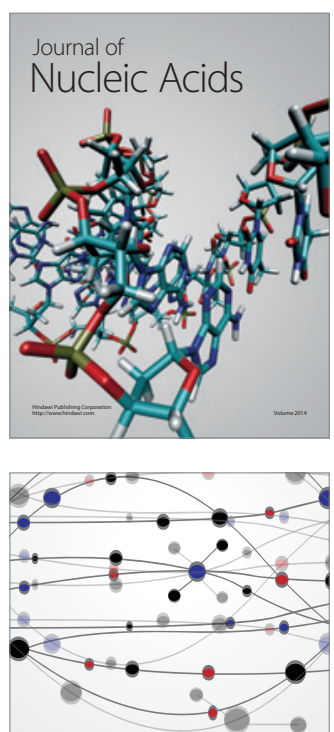

The Scientific World Journal
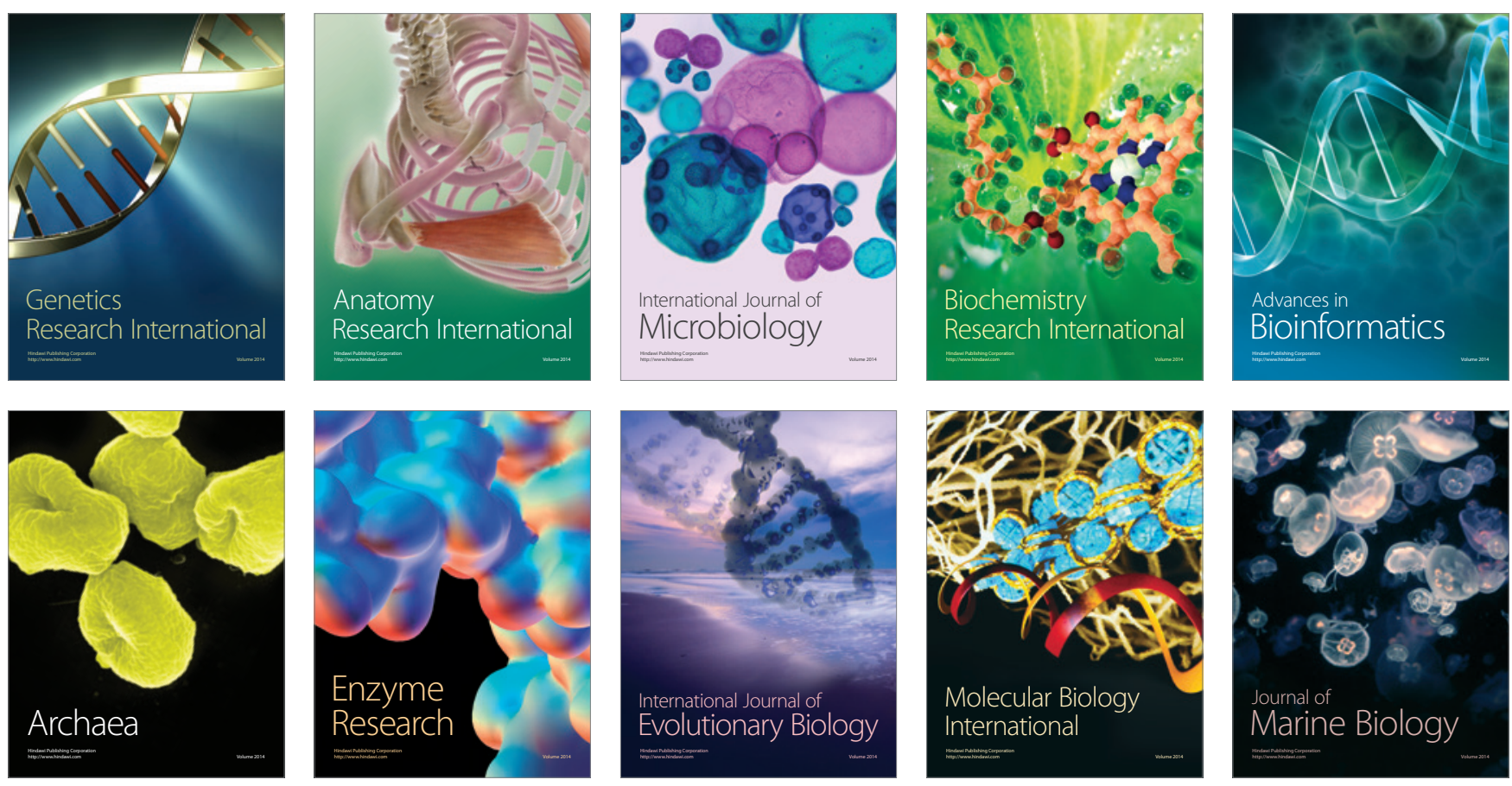